\title{
Clinical and molecular characterization of four patients with NTCP deficiency from two unrelated families harboring the novel SLC10A1 variant c.595A >C (p.Ser199Arg)
}

\author{
HUA LI $^{1}$, MEI DENG ${ }^{1}$, LI GUO $^{1}$, JIAN-WU QIU ${ }^{1}$, GUI-ZHI LIN ${ }^{1}$, \\ XIAO-LING LONG ${ }^{2}$, XIAO-MIN XIAO ${ }^{3}$ and YUAN-ZONG SONG ${ }^{1}$ \\ ${ }^{1}$ Department of Pediatrics, The First Affiliated Hospital of Jinan University, Guangzhou, Guangdong 510630; \\ ${ }^{2}$ Department of Pediatrics, Bo-Ai Hospital of Zhongshan, Zhongshan, Guangdong 528400; \\ ${ }^{3}$ Department of Gynecology and Obstetrics, The First Affiliated Hospital of Jinan University, \\ Guangzhou, Guangdong 510630, P.R. China
}

Received April 7, 2019; Accepted August 30, 2019

DOI: $10.3892 / \mathrm{mmr} .2019 .10763$

\begin{abstract}
Sodium taurocholate cotransporting polypeptide (NTCP), a carrier protein encoded by solute carrier family 10 member 1 (SLC1OA1), is expressed in the basolateral membrane of hepatocytes, where it is responsible for the uptake of bile acids from plasma into hepatocytes. The first patient with NTCP deficiency was described in 2015. A limited number of such patients have been reported in the literature and their genotypic and phenotypic features require further investigation. The current study investigated 4 patients with NTCP deficiency from two unrelated families. The patients were subjected to $S L C 10 A 1$ genetic analysis and it was revealed that all patients were compound heterozygous for the c.800C $>\mathrm{T}$ (p.Ser267Phe) and c.595A >C (p.Ser199Arg) SLC10Al variants . To the best of the authors' knowledge, the latter variant had not been previously reported. Further analysis in 50 healthy individuals did not identify carriers. The c.595A>C (p.Ser199Arg) variant exhibited co-segregation with hypercholanemia and exhibited a relatively conserved amino acid when compared with homologous peptides. Moreover, SWISS-MODEL prediction revealed that the mutation affected the conformation of the NTCP molecule. The 4 patients demonstrated varying degrees of hypercholanemia while a downward trend in the plasma levels of total bile acids (TBA) in 2 pediatric patients and occasionally normal TBA level in an adult case were observed. The results indicated an autosomal recessive trait for NTCP deficiency, supported the primary role of NTCP in the uptake of bile acids from plasma and suggested that hepatic uptake
\end{abstract}

Correspondence to: Professor Yuan-Zong Song, Department of Pediatrics, The First Affiliated Hospital of Jinan University, 613 Huangpu Da Dao Xi, Guangzhou, Guangdong 510630, P.R. China E-mail: songyuanzong@vip.tom.com

Key words: sodium taurocholate cotransporting polypeptide, solute carrier family 10 member 1, c.595A >C (p.Ser199Arg), hypercholanemia of bile acids may occur by means other than NTCP uptake. Moreover, the novel missense variant c.595A >C(p.Ser199Arg) enriched the $S L C 10 A 1$ mutation spectrum and may serve as a new genetic marker for the molecular diagnosis and genetic counseling of NTCP deficiency.

\section{Introduction}

Sodium taurocholate cotransporting polypeptide (NTCP) deficiency is an inborn error of bile acid metabolism caused by biallelic mutations of the solute carrier family 10 member 1 (SLC10A1) gene. SLC10A1 is located in chromosome 14q24.2, contains 5 exons and has a total length of $23 \mathrm{~kb}(1,2)$. The protein product NTCP is composed of 349 amino acid residues and has a molecular weight of $38 \mathrm{kDa}$ (1). NTCP is a transporter protein expressed in the sinusoidal plasma membrane of hepatocytes, where it is involved in the uptake of bile salts from plasma into hepatocytes in a sodium-dependent manner, playing a crucial role in the enterohepatic circulation of bile acids $(3,4)$.

The SLC10Al gene was cloned by Hagenbuch et al (1) in 1994 and the function of NTCP has since been widely investigated $(5,6)$. Theoretically, NTCP deficiency was thought to cause hypercholanemia (7); however, there was no clinical description of such patients until 2015, when Vaz et al (8) reported the first patient with NTCP deficiency (8). At the time of publication of the current study, only a limited number of patients with such a condition have been reported in 10 publications (8-17). The genotypic and phenotypic features of this newly described condition therefore require further investigation.

The reported SLC10A1 variants causing NTCP deficiency included c.755G $>$ A(p.Arg252His) (8), c.615_618del (p.Ser206Profs*12) (9), c.263T >C(p.Ile88Thr) (10) and c.800C $>$ T(p.Ser267Phe) (12). Among them, the variant p.Ser267Phe is rather prevalent, with the allele frequency of 7.4\% (23/312), 3.1\% (9/294) and 9.2\% (28/306) in Chinese, Korean and Vietnamese populations (6), respectively. Therefore, although patients with NTCP deficiency were rarely reported in the past over 20 years, this condition may not be rare, especially in East Asian population. The present 
study described the identification of 4 new patients with NTCP deficiency in 2 unrelated families and presented the clinical and genetic findings of the aforementioned patients.

\section{Materials and methods}

Patients and ethical approval. A total of 2 pediatric patients, including a 27.7-month-old male and a 3.8-month-old female, from two unrelated families and 2 adult patients, including a male aged 31 years and a female aged 35 years, from family 1 with hypercholanemia as well as their family members were included in the current study, which was performed between May 2016 and July 2017 in The First Affiliated Hospital of Jinan University. The clinical findings were collected and described as case reports in the Results section of the present study. In order to explore the allele frequency of the identified novel SLC10Al variant, 50 blood samples (1-2 $\mathrm{ml}$ in volume for each sample, with a total of 100 SLC10A1 alleles) from healthy volunteers were collected to serve as the controls.

The current study was approved by the Committee for Medical Ethics, The First Affiliated Hospital of Jinan University and written informed consent was obtained from the parents of the patients and all the healthy controls.

Sanger sequencing. Genomic DNA was extracted using a DNA extraction kit (Simgen) according to the manufacturer's protocol. The five SLC1OAl exons and their flanking sequences, including the 5'- and 3'-untranslated regions as well as 309 base pairs upstream of the transcriptional start site, were amplified by PCR as described previously (10). The PCR products were purified using a gel extraction kit (Omega Bio-Tek, Inc.) and Sanger sequencing was subsequently performed on a 96-capillary ABI 3730x1 DNA Analyzer (Applied Biosystems; Thermo Fisher Scientific, Inc.) with a BigDye Terminator v 3.1 Cycle Sequencing kit (Thermo Fisher Scientific, Inc.).

The sequencing results were aligned with the SLC1OAI gene sequence, which was available at Ensembl Genome Browser (www.ensembl.org), using DNAman software (version 5.2.2; Lynnon Biosoft Corporation) and analyzed using Chromas software (version 2.6.6; Technelysium Pty, Ltd.). The allele frequency of the identified novel SLC10Al variant was investigated in four population databases, including the 1000 Genomes Project (browser.1000genomes.org), the Exome Sequencing Project (esp.gs.washington.edu/drupal), the Exome Aggregation Consortium (exac.broadinstitute.org) and the Human Gene Mutation Database (www.hgmd.cf.ac. uk/ac/index.php).

PCR-restriction fragment length polymorphism (RFLP) approach. In addition to the PCR-RFLP approach described previously to detect the pathogenic SLC10A1 variant c.800C $>\mathrm{T}$ (p.Ser267Phe) (12), a novel PCR-RFLP procedure was developed in the present study to verify the SLC10AI genotypes of the patient family members and to screen for the novel SLC10Al variant in 50 healthy individuals. The nucleotide sequences of the forward and reverse primers used in PCR were 5'-CCACCTCTGTTCCTCTCTATCC-3' and 5'-GCAACAGAGTGAGACCCTTTC-3', respectively (Invitrogen; Thermo Fisher Scientific, Inc.). The target fragment was amplified using a PCR kit (Takara Biotechnology
Co., Ltd.) and the PCR thermocycling conditions were: $94^{\circ} \mathrm{C}$ for $5 \mathrm{~min}$, followed by 35 cycles at $94^{\circ} \mathrm{C}$ for $30 \mathrm{sec}, 58^{\circ} \mathrm{C}$ for $40 \mathrm{sec}$ and $72^{\circ} \mathrm{C}$ for $50 \mathrm{sec}$, and $72^{\circ} \mathrm{C}$ for $10 \mathrm{~min}$. The HpyCH4V restriction enzyme (Thermo Fisher Scientific, Inc.) was used to digest the PCR products and the digested DNA products were subsequently separated by electrophoresis in a $4 \%$ agarose gel. For frequency calculation of the novel variant, the number of mutated alleles detected in all 50 control samples was divided by 100 and the quotient was multiplied by $100 \%$.

Alignment of homologous peptides. The amino acid sequences for the peptides homologous to human NTCP were identified in 99 species using the Ensembl Genome Browser. The 99 species were then classified into five taxonomy subgroups: Primates (24 species), rodents and lagomorphs (26 species), other mammals (24 species), other vertebrates (21 species) and other species (4 species), and aligned using BLAST/BLAT Ensembl software (Ensembl Release 97, July 2019: www. ensembl.org/Multi/Tools/Blast?db=core).

In silico prediction of pathogenicity. A total of four prediction programs were used to predict the pathogenicity of the novel SLC10Al variant. PolyPhen-2 (genetics.bwh.harvard. edu/pph2) analysis identifies a variant as 'probably damaging' if the probability is $>0.85$ and as 'possibly damaging', if the probability $>0.15$ (18). MutationAssessor (mutationassessor. org) scores a mutation by global and subfamily specific conservation patterns as low, medium or high (19). Sorting Intolerant From Tolerant (SIFT; sift.jcvi.org) classifies the variant as being 'deleterious' if the prediction score is $<0.05$ (20). Moreover, the deleterious annotation of genetic variants using neural networks (DANN; cbcl.ics.uci.edu/public_data/DANN) may be used to annotate the pathogenicity of genetic variants using neural networks and higher values are associated with deleterious variants (21).

Effect of the novel mutation on the structure of the NTCP protein. The wild structural model of NTCP protein was built using the online software SWISS-MODEL automated protein modeling server (swissmodel.expasy.org). The tertiary structures of the wild type and mutant NTCP protein were compared by SWISS-Pdb Viewer 4.1.0 (www.Expasy. org/spdbv) to evaluate the effect of the variant on structure of the NTCP molecule.

\section{Results}

Patient 1. A 27.7-month-old male was referred to The First Affiliated Hospital of Jinan University due to sustained elevated serum total bile acids (TBA). A health examination 1.2 months prior to admission revealed that the patient had a TBA level of $103.8 \mu \mathrm{mol} / 1$ and elevated transaminases. A biochemistry test repeated 3 weeks after the initial test revealed persistent hypercholanemia and elevated aspartate transaminase level (Table II). The patient was subsequently referred to the First Affiliated Hospital of Jinan University for further investigation and management.

The patient had frequent early fetal heart decelerations that were detected by cardiotocography during labor at the 


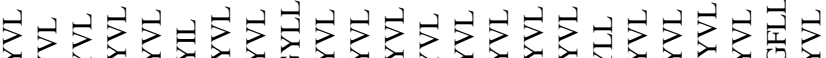

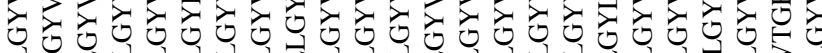

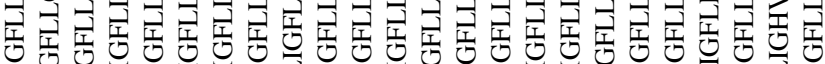

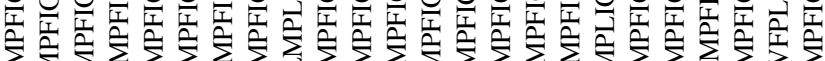

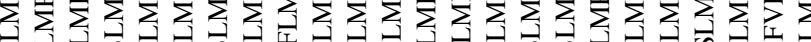

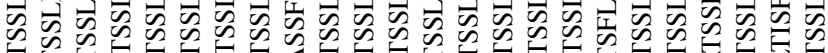

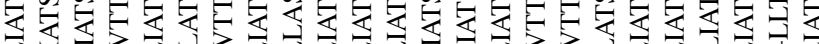

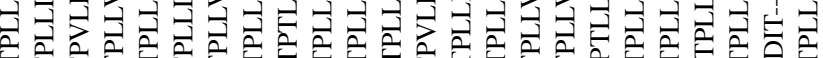

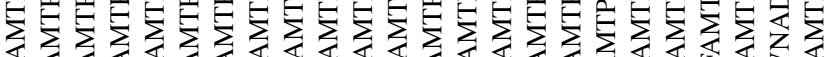

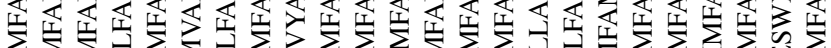

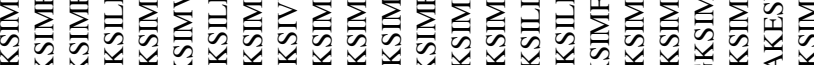

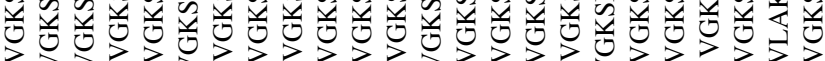

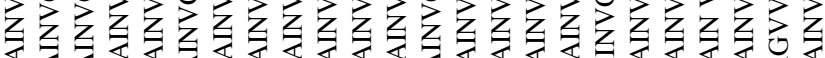

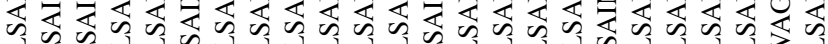

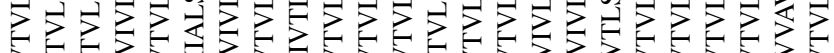

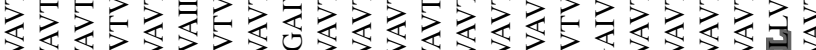

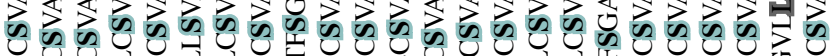

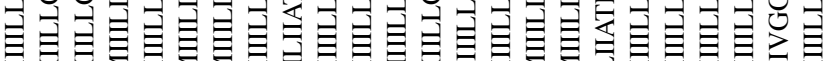

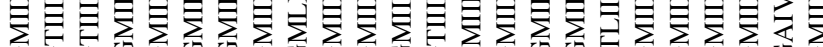
U్

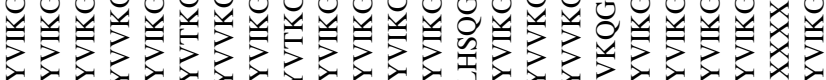

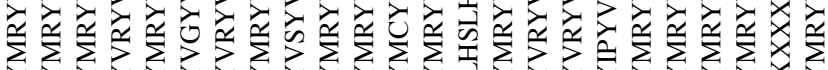

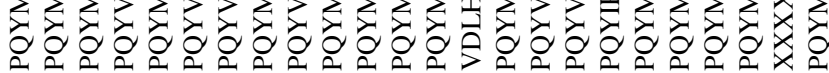

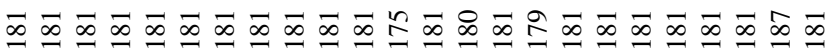

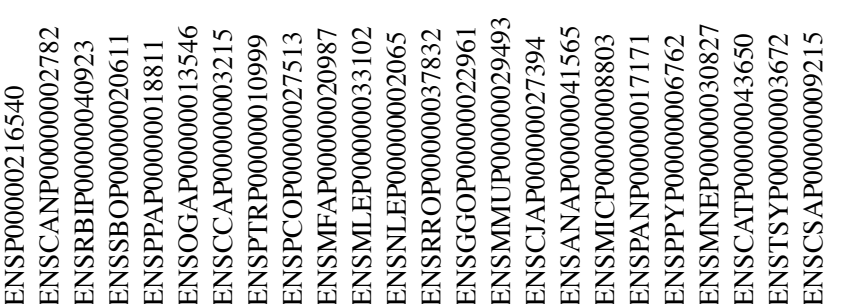

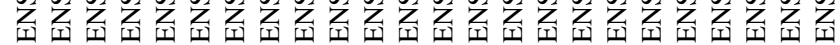

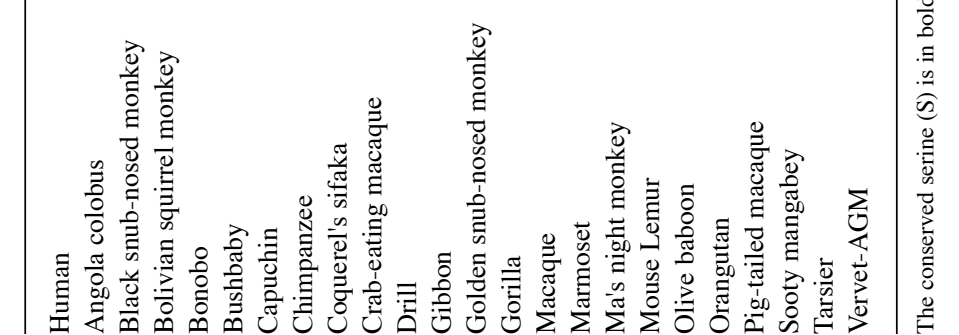




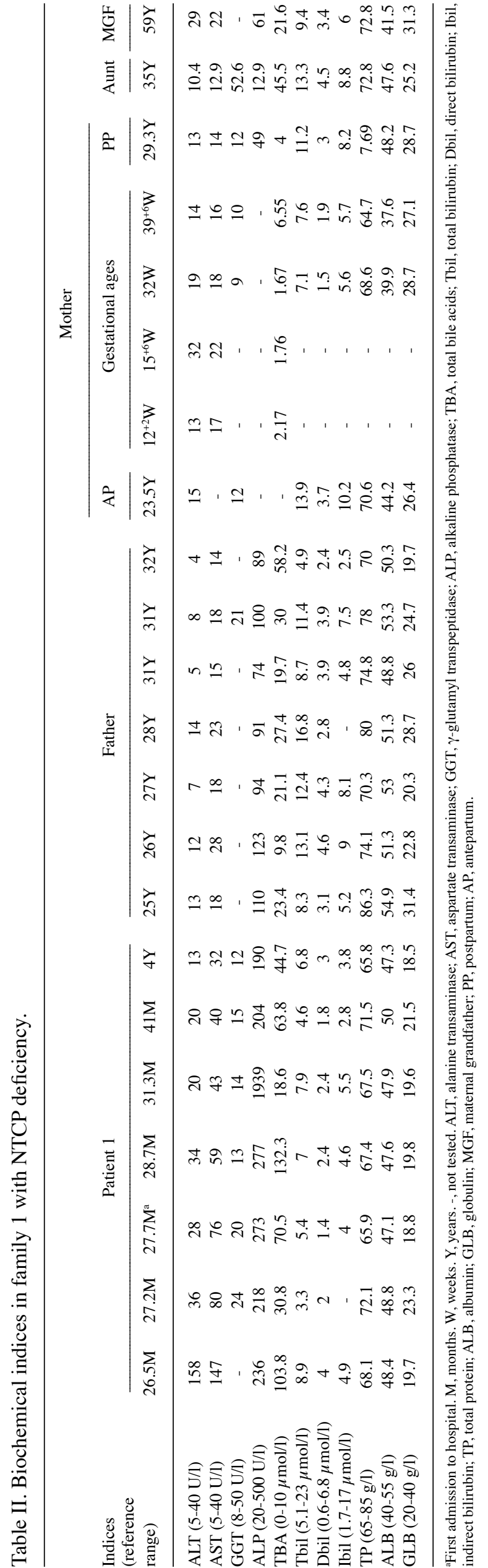

gestational age of 39 weeks and 5 days (Fig. 1) and the patient was subsequently delivered by a cesarean section. The patient had a birth weight of $3.0 \mathrm{~kg}$ and a body length of $47 \mathrm{~cm}$. The patient's mother was healthy during and after pregnancy; however, the father had occasional hypercholanemia without any clinical manifestations. Additionally, the patient's paternal aunt exhibited raised TBA levels without any clinical symptoms. The TBA levels in the paternal grandparents and maternal grandmother were in the normal range. The maternal grandfather exhibited slightly elevated levels (Table II) and a liver ultrasonogram revealed a $5 \times 4 \mathrm{~mm}$ hemangioma in the right lobe.

A physical examination revealed that the patient had a body weight of $11.5 \mathrm{~kg}$, height of $85.5 \mathrm{~cm}$ and a head circumference of $49 \mathrm{~cm}$. No dysmorphic appearance or jaundice were observed in the skin and sclera. No stridor, crackles or crepitus were heard following auscultation of the lungs. The heart sounds were normal without any murmurs. The liver and spleen were nonpalpable. The tone of the body and limbs appeared normal.

Biochemical analysis of the patient revealed that the levels of TBA and transaminase were elevated (Table II). The SLC1OAl gene was analyzed to evaluate the possibility of NTCP deficiency. Thereafter, no special treatment was administered to the patient except vitamin D supplementation. Subsequent clinic follow-ups revealed that the patient's aspartate transaminase gradually decreased to normal levels; however, serum TBA was persistently elevated and globulin levels exhibited an occasional decrease (Table II). The patient exhibited normal anthropometric social performance.

Patient 2. A 3.8-month-old female patient was referred to the First Affiliated Hospital of Jinan University with increased TBA levels that had persisted for 3.5 months. The patient was admitted into the neonatal unit in the First Affiliated Hospital of Jinan University as a preterm infant. The infant was delivered by a cesarean section as the first-born of dichorionic diamniotic twins at the gestational age of 32 weeks and 3 days (birth weight, $1.4 \mathrm{~kg}$; body length, $40 \mathrm{~cm}$ ). Her dizygotic twin sister, who had a birth weight of $1.85 \mathrm{~kg}$ and body length of $45 \mathrm{~cm}$, exhibited occasional hypercholanemia (Table III).

The patient's parents were healthy and there was no family history of genetic diseases. The patient had a slightly increased serum TBA levels 9 days following birth. Additionally, the patient developed hypoalbuminemia during hospitalization and was treated with intravenous albumin. The patient was discharged with unresolved hypercholanemia 25 days following birth. The elevation of TBA was intractable on subsequent clinic follow-up, with a peak level $260.5 \mu \mathrm{mol} / \mathrm{l}$ at the age of 2.3 months (Table III).

Physical examination revealed a body weight of $4.7 \mathrm{~kg}$, height of $54 \mathrm{~cm}$ and a head circumference $36.5 \mathrm{~cm}$. There was no dysmorphic appearance or jaundice in the skin and sclera. The skull and facial appearance were not malformed. No positive signs were found in the lungs and the heart. The patient's liver and spleen were not enlarged. The tone of the body and limbs appeared normal. Biochemical analysis revealed elevated TBA levels, with a peak of $256.5 \mu \mathrm{mol} / 1$ and occasional decreases in globulin and albumin levels. All other indices were normal (Table III). 

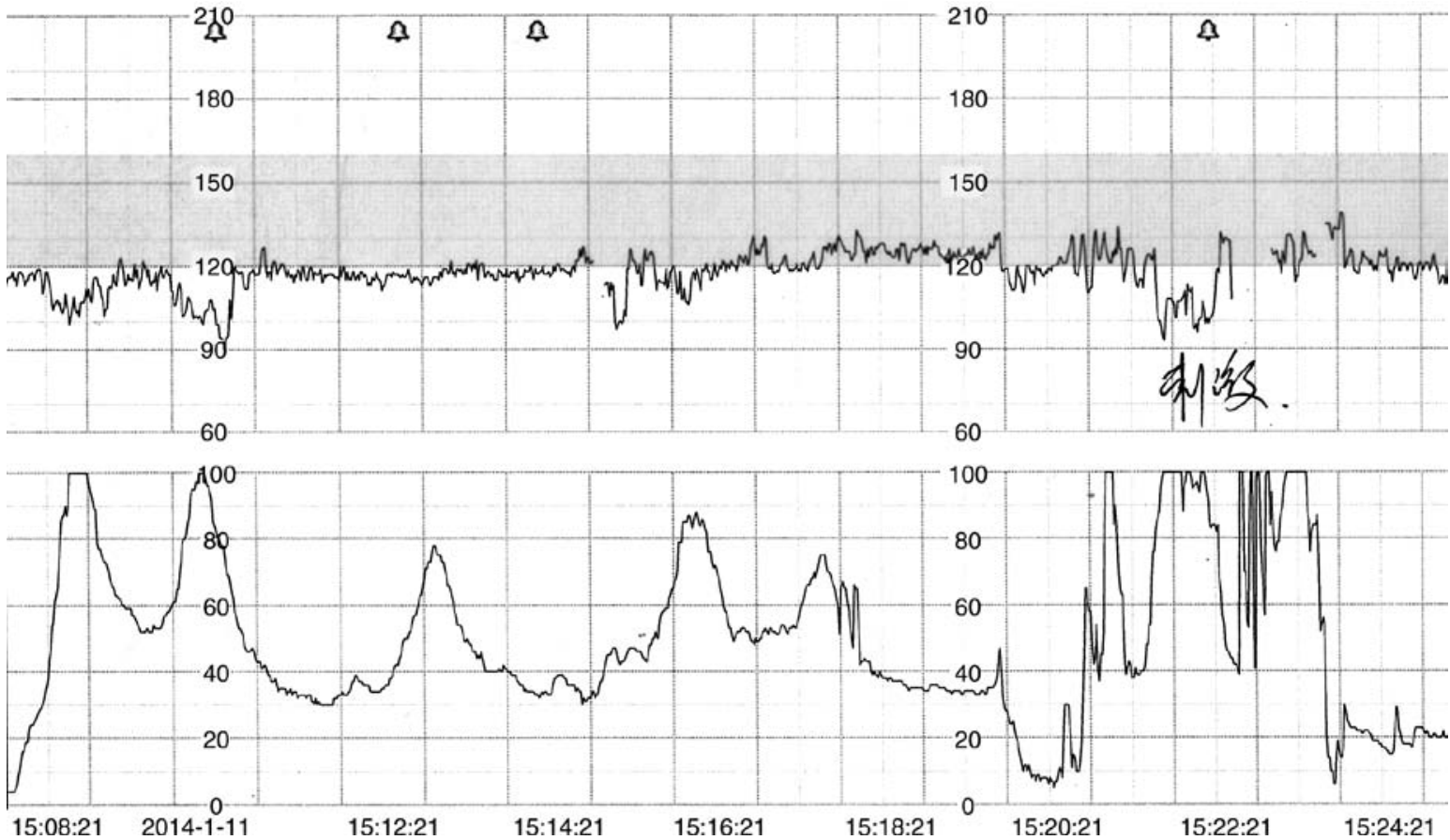

Figure 1. Cardiotocogram obtained during the delivery of patient 1 . The upper curve recorded fetal heart rate while the lower curve recorded uterine contractions. The two decelerations on the left, with the lowest rate of 90 beats per minute, were relieved spontaneously when uterine contractions ceased. The deceleration on the right was caused by vaginal examination.

As the patient exhibited persistent hypercholanemia, SLC10A1 analysis was performed to evaluate the possibility of NTCP deficiency. No special treatment other than vitamin D and zinc supplements was given thereafter. The patient was followed-up to the age of 9.1 months and exhibited normal development patterns. During the follow-up, the patient had persistently elevated TBA levels, while other liver indices were in the normal range (Table III).

SLC10A1 genotypes. Sanger sequencing of the SLC10A1 gene in the two families demonstrated that both index patients were compound heterozygotes for the variants c. $800 \mathrm{C}>\mathrm{T}(\mathrm{p}$. Ser267Phe) and c.595A >C(p.Ser199Arg). In family 1, the father shared the same genotype with patient 1 while the mother was a carrier of the variant $800 \mathrm{C}>\mathrm{T}$ (p.Ser267Phe) (Fig. 2A). In family 2 , the father was a carrier of the variant c.595A $>C(p$. Ser199Arg), while the mother was a carrier of $c .800 C>T(p$. Ser267Phe) (Fig. 2B). To the best of our knowledge, the variant c.595A >C (p.Ser199Arg) has not been previously reported by other studies in the PubMed database (www.ncbi.nlm. nih.gov) and is not currently included in the 1000 Genomes Project (browser.1000genomes.org), the Exome Sequencing Project (esp.gs.washington.edu/drupal), and the Human Gene Mutation Database (www.hgmd.cf.ac.uk/ac/index.php). The Exome Aggregation Consortium (exac.broadinstitute.org) database has recorded the variant c.595A >C (p.Ser199Arg), with an allele frequency of $0.3 \%$ in East Asian populations and of $0.025 \%$ in all humans.

PCR-RFLP findings. A novel PCR-RFLP approach was developed to explore the SLC1OAl genotypes in the two families and to investigate the frequency of the novel variant c. $595 \mathrm{~A}>\mathrm{C}$ (p.Ser199Arg) in healthy controls. As presented in Fig. 3, in addition to the 3 patients diagnosed by Sanger sequencing, the patient's aunt in family 1 was also affected by NTCP deficiency, having an SLC10Al genotype of c.800C $>$ T(p.Ser267Phe)/c.595A $>$ C(p.Ser199Arg). Moreover, no c.595A $>$ C(p.Ser199Arg) carrier status was detected in 50 healthy controls, indicating an allele frequency of $<1 \%$. Therefore, the missense variant identified in the present study was a novel SLC10A1 mutation and not a single nucleotide polymorphism.

Bioinformatics analysis. The amino acid sequences of the homologous peptides in a total of 99 species were aligned to allow comparative analysis. Although the amino acid p.Ser199 was not conserved in other vertebrates (11/21) and other species (1/4) (data not shown), this residue was relatively conserved in primates and mammals. In fact, this residue was found in 23/24 of the primates including humans (Table I), 21/26 of rodents and lagomorphs and 16/24 of the other mammals (data not shown).

In silico tools were subsequently used to predict the pathogenicity of the novel mutation. Polyphen 2 and DANN generated the same outcome ('possibly damaging'), with a prediction score of 0.977 and 0.986 , respectively. MutationAssessor yielded a result of 'medium' with a prediction score of 3.07, while SIFT suggested that the mutation was 'tolerated', with a prediction score of 0.08 .

SWISS-MODEL software was used for structural prediction of the NTCP protein. The c.595A >C (p.Ser199Arg) variant resulted in the replacement of a serine by an arginine at the amino acid position 199 of the NTCP molecule. Arginine produced a new hydrogen bond with the proline at position 286. Additionally, the hydrogen bond distances between specific amino acids in the NTCP protein were altered (Fig. 4), distorting the molecular structure of the NTCP protein. 


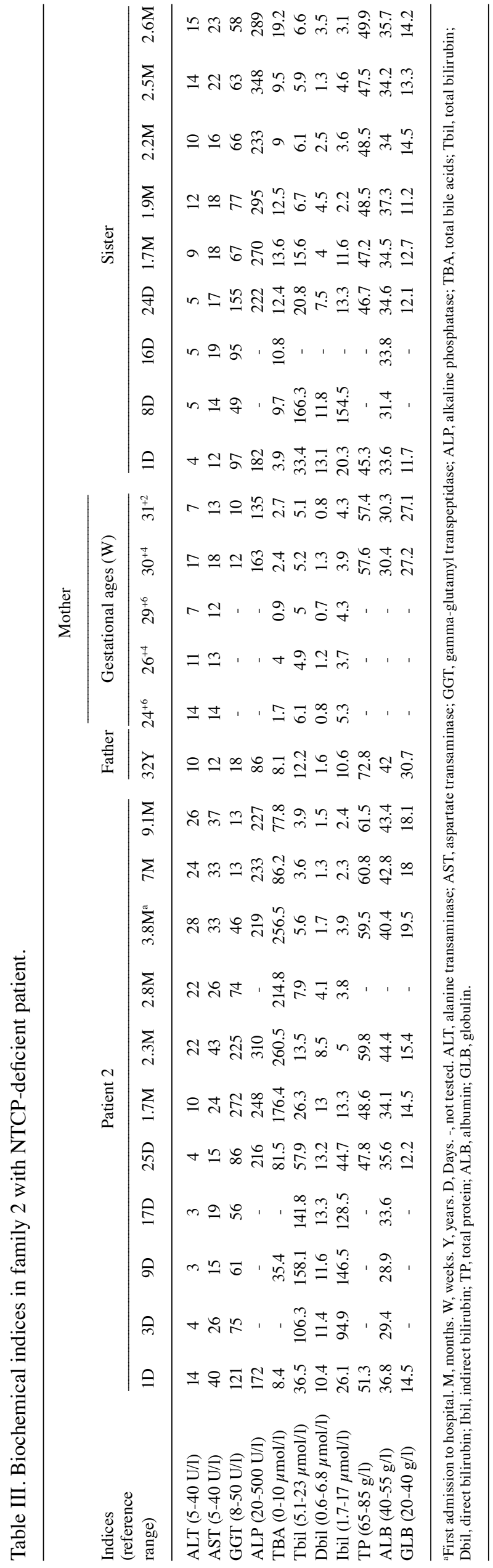

\section{Discussion}

The present study identified 4 patients with NTCP deficiency, including 2 pediatric and 2 adult individuals. Analysis revealed that the patients were compound heterozygous for the $S L C 10 \mathrm{Al}$ variants c.800C $>\mathrm{T}$ (p.Ser267Phe) and c.595A $>\mathrm{C}$ (p.Ser199Arg). The former variant has previously been reported to be pathogenic by functional and bioinformatics approaches and clinical studies $(6,9,12-16)$. The latter unreported variant demonstrated a frequency of $<1 \%$ in 50 healthy individuals in the present study and was absent from controls in the 1000 Genomes Project, the Exome Sequencing Project and the Human Gene Mutation Database. Exome Aggregation Consortium database has recorded the variant c.595A>C (p.Ser199Arg); however, this database is not based on findings on patients but general populations and the low allele frequencies of this variant in East Asian $(0.3 \%)$ and all humans $(0.025 \%)$ indicated a mutation, constituting supporting evidence of pathogenicity. The c.595A $>C(p$. Ser199Arg) mutation exhibited co-segregation with hypercholanemia and was detected in trans with the c.800C $>\mathrm{T}(\mathrm{p}$. Ser267Phe) variant in 4 patients from two unrelated families. Moreover, this novel mutation exhibited a conserved amino acid and distorted the conformation of the NTCP molecule. While functional analysis of the novel SLC1OAl variant was not performed in the current study due to technical limitations, the results obtained supported the diagnosis of NTCP deficiency in the patients investigated. Particularly, the functional study constituted a work plan for the authors' future investigation, since it provides much stronger evidence of pathogenicity. Moreover, the present group has been committed to the clinical diagnosis of NTCP deficiency since the first pediatric patient in China and the first adult worldwide with such a condition were reported in 2016 (12), but unfortunately no additional patients harboring the novel SLC10A1 variant c.595A >C (p.Ser199Arg) were diagnosed, suggesting that this variant was not so prevalent in Chinese populations.

In silico tools were used to predict the pathogenicity of the SLC10A1 variant c.595A>C(p.Ser199Arg). Polyphen 2 and DANN predicted pathogenic outcomes, and this might be considered as computational evidence supporting the deleterious effect on the SLC10A1 gene, according to the American College of Medical Genetics and Genomics standards and guidelines for the interpretation of sequence variants (22). However, Mutation Assessor and SIFT generated inconsistent results. This discrepancy therefore requires further investigation as the accuracy of prediction algorithms is affected by a number of variables, including the gene examined, the number of sequences in the alignment, the evolutionary distances among species and the importance of absolute amino acid conservation vs. relatively conservative missense changes (23). In general, the accuracy of the majority of algorithms used for missense variant prediction is $\sim 65-80 \%$, even when applied to established disease-causing variants (22). As clinical data describe human disease more directly, clinical observations should be considered more persuasive when a discrepancy or conflict arises between clinical and functional observations (24).

The 4 patients in the current study presented with persistent hypercholanemia and supported the primary role of NTCP in the uptake of bile acids from plasma. However, the plasma 
A

Patient 1

Father

Mother

TTGTGCCGTGTGGTTGTGCCGTGTGGC TTGTGCAGTGTGGC

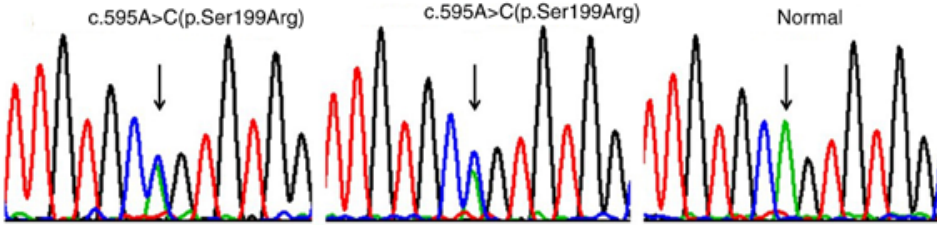

TCTGTTCCACCAT TCTGTTCCACCAT TCTGTTCCACCAT

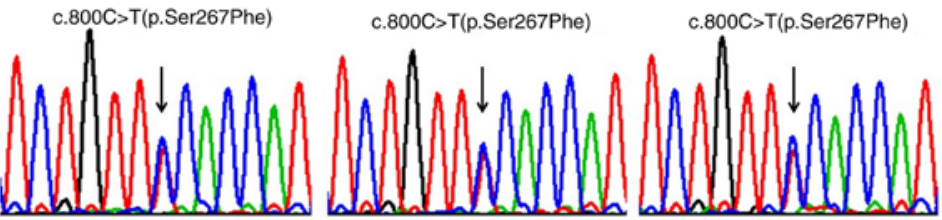

B

Patient 2

Father

Mother

CTTGTGCCGTGTGGC CTTGTGCCGTGTGGC TTGTGCAGTGTGGC
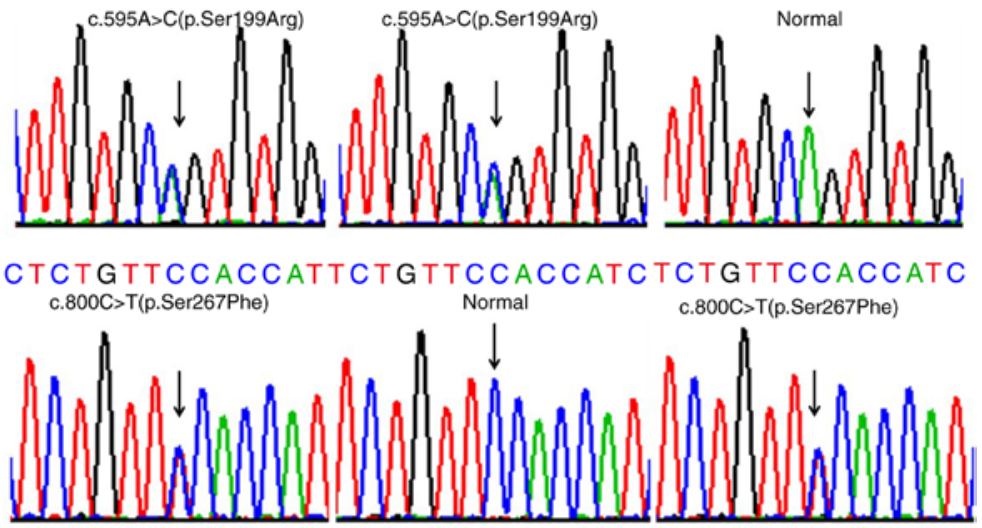

Figure 2. Sanger sequencing results of $S L C 10 A 1$ gene in the two families. (A) Patient 1 and his father were heterozygous for the $S L C 10 A 1$ variants c.800C $>\mathrm{T}$ (p.Ser267Phe) and c.595A >C(p.Ser199Arg) while his mother was a carrier of variant c.800C $>\mathrm{T}(\mathrm{p}$.Ser267Phe). (B) Patient 2 was a compound heterozygote of variants c.800C $>$ T(p.Ser267Phe) and c.595A $>$ C(p.Ser199Arg) while her father was a carrier of c.595A $>$ C and her mother was a carrier of c.800C $>$ T. SLC10A1, solute carrier family 10 member 1 .
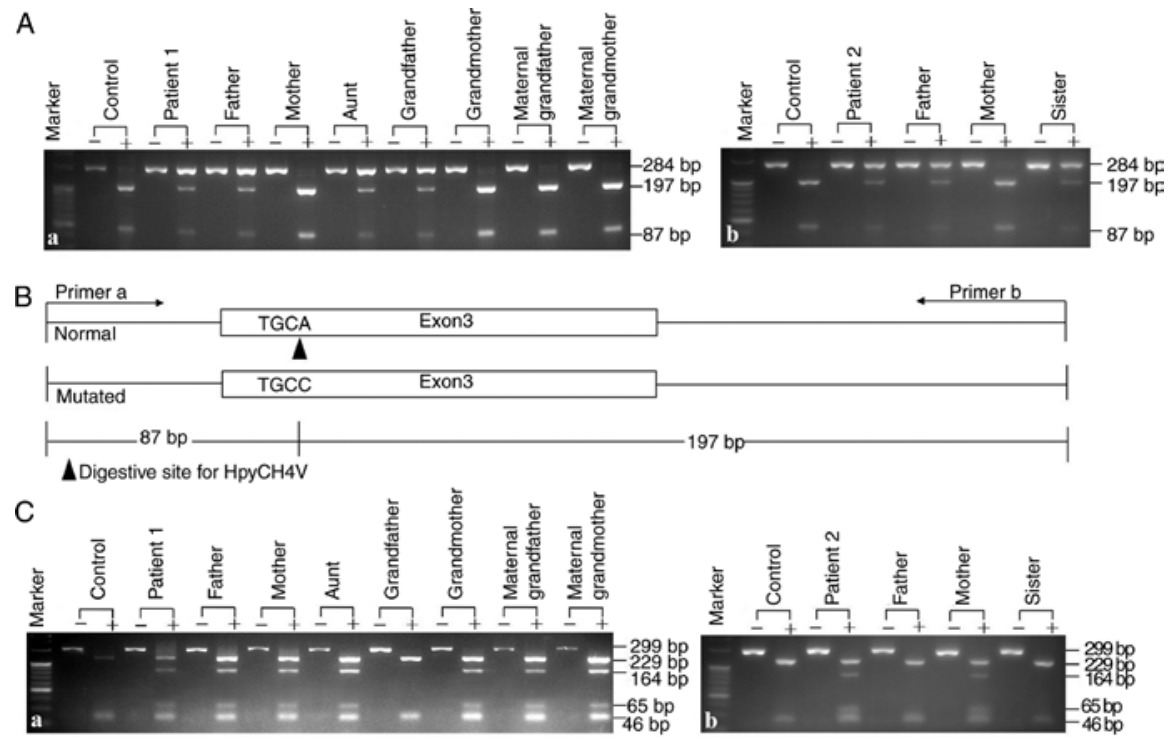

Figure 3. PCR-RFLP protocols screening for the variants c.800C $>\mathrm{T}$ (p.Ser267Phe) and c.595A $>\mathrm{C}(\mathrm{p}$. Ser199Arg). Representative gel electrophoresis images of the digested products by the newly-developed PCR-RFLP protocol for the detection of the novel variant c.595A>C(p.Ser199Arg) in family (Aa) 1 and (Ab) 2 . The results showed that patient 1, his father, aunt and grandfather and patient 2, her father and sister all harbored the c.595A $>$ C(p.Ser199Arg) variant. (B) A schematic diagram of the PCR-RFLP approach. The wild-type SLC10A1 allele had a HpyCH4V restriction enzyme site and produced the 87 and 197 bp fragments from the $284 \mathrm{bp}$ band following enzymatic digestion. A representative gel electrophoresis image for PCR-RFLP screening for the c.800C $>\mathrm{T}$ (p.Ser267Phe) variant in family $1(\mathrm{Ca})$ and $2(\mathrm{Cb})$. The results showed that patient 1 , his father, mother, aunt, grandmother, maternal grandfather and maternal grandmother and patient 2 and her mother harbored the c.800C $>$ T(p.Ser267Phe) variant. RFLP, restriction fragment length polymorphism; SLC10A1, solute carrier family 10 member 1 . 


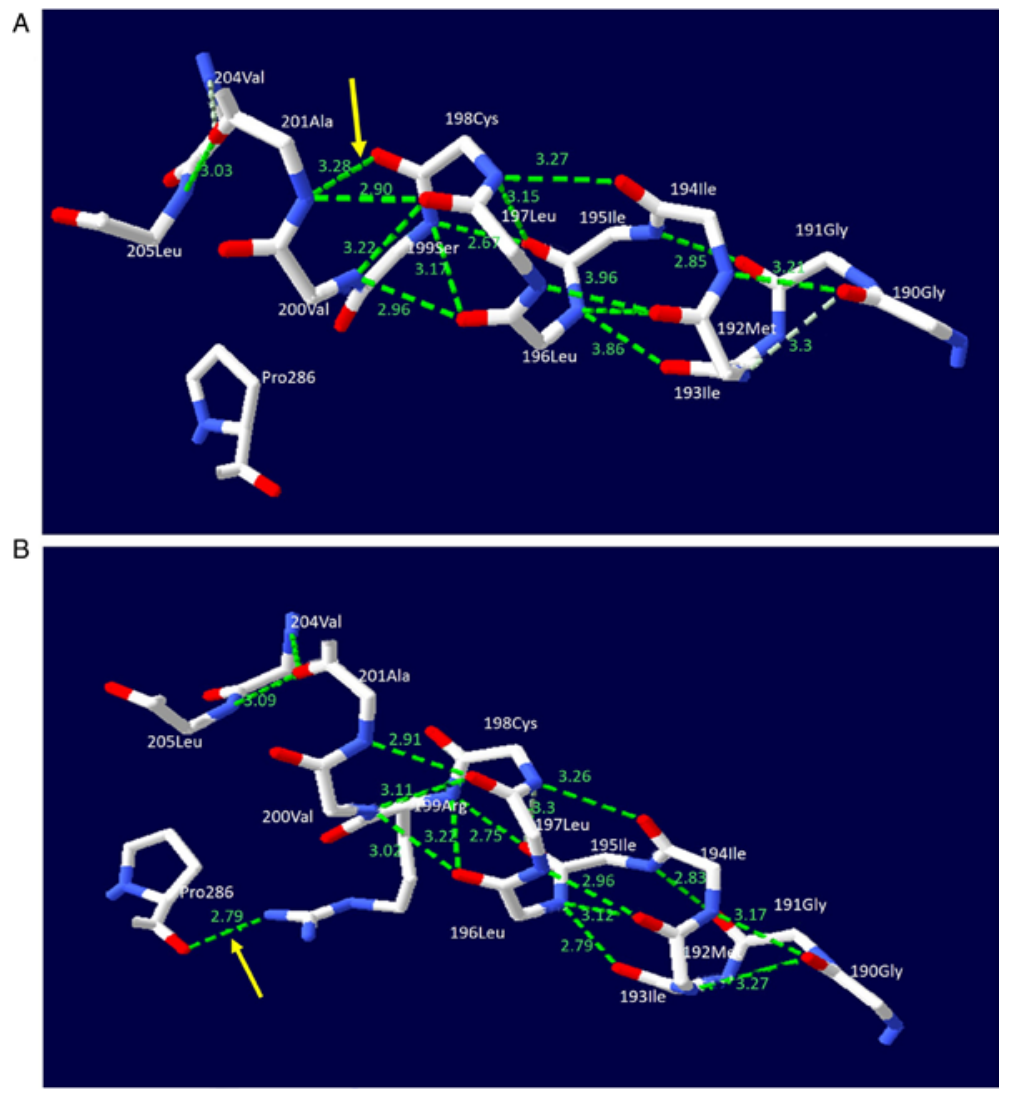

Figure 4. NTCP molecular alteration caused by the solute carrier family 10 member 1 variant c.595A>C(p.Ser199Arg). The figure illustrates a ball-and-stick model of the human NTCP protein. The solid lines in white, red and blue represent carbon, oxygen and nitrogen atoms, respectively, while the dashed lines in green represent hydrogen bonds. (A) In the wild-type model, the serine at position 199 in NTCP was not associated with the proline at position 286. (B) In the mutant NTCP model, the serine at position 199 was changed to an arginine. This change led to the formation of a new hydrogen bond between the arginine and proline (denoted by the yellow arrow). Meanwhile, the hydrogen bond lengths between the Gly at 190 and the Ile at 194, the Gly at 190 and Ile at 193, the Gly at 191 and Ile at 195, the Met at 192 and Leu at 196 as well as the Ile at 195 and Cys at 198, were changed from 3.17, 3.3, 2.85, 2.86 and 3.15 ̊ to 3.21, 3.27, 2.83, 2.79 and $3.3 \AA$, respectively. The changes in the hydrogen bonds resulted in structural distortion of the normal NTCP molecule. NTCP, sodium taurocholate cotransporting polypeptide.

TBA levels tended to decrease over time in the two pediatric patients and the father of patient 1 exhibited an occasionally normal TBA level. The aforementio-ned trends suggested that mechanisms other than NTCP are involved in the hepatic uptake of bile acids. Solute carrier organic anion transporter family member $1 \mathrm{~B} 1$ and $3(O A T P 1 B 1 / 3)$, two members of the organic anion transporting polypeptides family, are heterodimers expressed in the basal membrane of hepatocytes and clear bile acid salts such as cholic acid, glycolic acid and taurocholic acid from the plasma (25). OATP1B1 and OATP1B3 demonstrate an age-dependent maturation process in humans and it has been reported that their hepatic expression in all pediatric age groups was significantly lower compared with adults (26). Furthermore, the expression level of OATPI at birth is $15 \%$ of adult levels (25). Additionally, the organic solute transporter $\alpha / \beta$ is a heterodimer expressed in the membrane of hepatocytes, where it functions as a bidirectional organic solute transport system that transports bile acids (27). The farnesoid X receptor is activated by bile acids (28) and subsequently inhibits the synthesis of bile acids in hepatocytes and upregulates the expression of organic solute transporters $\alpha$ and $\beta(\mathrm{OST} \alpha / \beta)$ to remove bile acids from the plasma (29-31). The presence of the aforementioned maturation and regulation mechanisms suggests that the bile acid levels in patients with NTCP deficiency may not increase uncontrollably, may decline with age and may approach normal as observed in the father of patient 1 in the current study.

The hypoglobulinemia observed in patients 1 and 2 and the hypoalbuminemia seen in patient 2 may be a transient alteration associated with their age and not related to NTCP deficiency. Globulin is closely associated with immune function (32), which gradually develops and matures in children (33). Moreover, hypoalbuminemia is associated with immature liver synthesis function in children, particularly preterm infants (34) and may result in the gradually-corrected hypoalbuminemia observed in patient 2 .

The patients with NTCP deficiency reported in previous studies were homozygous or compound heterozygous for SLC10A1 variants, indicating an autosomal recessive disorder (8-14). In the current study, however, the maternal grandfather of patient 1 and the sister of patient 2, were carriers of the variant c.800C $>\mathrm{T}$ (p.Ser267Phe) and c.595A $>C$ (p.Ser199Arg) respectively and exhibited hypercholanemia. In the former case, the specific cause for the elevated TBA level was unknown but may be attributed at least in part to hepatic hemangioma. In the latter case, a reasonable explanation may be physiological cholestasis, which results in temporarily high TBA levels in normal newborns and infants due to immature 
liver function (35-37). Moreover, her hyperbilirubinemia and high $\gamma$-glutamyl transpeptidase level may also reflect her immature liver function as a preterm infant. The NTCP protein is gradually expressed on the plasma membrane of hepatocytes in an age-dependent manner and its glycosylation is not completed until $\sim 1$ year after birth (38).

Patient 1 in the current study was delivered by cesarean section due to early fetal heart decelerations that were associated with uterine contractions and vaginal examination during labor. NTCP deficiency itself in the affected fetus may not be associated with the heart decelerations. Bile acids are synthesized in the fetus from 12 weeks of gestation (39) and are exported into the maternal circulation via the placenta $(40,41)$. Trophoblast cells in the placental barrier express bile acid carrier proteins, which are involved in the one-way transport of bile acids from the fetus to the mother (42). The mother of patient 1 had normal serum TBA levels during pregnancy, therefore fetal NTCP deficiency was unlikely to affect the fetus to cause early fetal heart decelerations during labor. Moreover, to the best of our knowledge, early fetal heart decelerations were not observed in 8 pediatric patients with NTCP deficiency with uncomplicated deliveries (8,10,12-15), suggesting that early fetal heart decelerations are not associated with NTCP deficiency.

In conclusion, by way of clinical and SLC10Al genetic analysis, a total of 4 new patients with NTCP deficiency from two unrelated families were diagnosed in the current study. The results obtained in the current study suggested an autosomal recessive trait for NTCP deficiency, supported the primary role of NTCP in the uptake of bile acids from plasma and suggested the presence of mechanisms of bile acid hepatic uptake other than NTCP. Moreover, the novel missense variant c.595A $>C$ (p.Ser199Arg) enriched the SLC10A1 mutation spectrum and constituted a new genetic marker for the molecular diagnosis and genetic counseling of NTCP deficiency.

\section{Acknowledgements}

The authors would like to thank Dr Shu Chen from the Department of Gynecology and Obstetrics of the First Affiliated Hospital of Jinan University for assisting with the analysis of the birth history of patient 1 .

\section{Funding}

The present study was supported by the National Natural Science Foundation of China (grant nos. 81570793, 81741080 and 81974057).

\section{Availability of data and materials}

The datasets used or analyzed during the present study are available from the corresponding author on reasonable request.

\section{Authors' contributions}

YZS, MD, JQ and LG conceived and designed the experiments. MD, JQ and LG performed the experiments. HL and XL collected the clinical data. YS, HL and XX analyzed the data. YS and HL wrote the manuscript.

\section{Ethics approval and consent to participate}

The current study was approved by the Committee for Medical Ethics, The First Affiliated Hospital, Jinan University, Guangzhou, China and adhered to the World Medical Association Declaration of Helsinki (2008). Written informed consent was obtained from the parents of the patients and all the healthy controls.

\section{Patient consent for publication}

Written informed consent was obtained from the parents of the patients and all the volunteers.

\section{Competing interests}

The authors declare that they have no competing interests.

\section{References}

1. Hagenbuch B and Meier PJ: Molecular cloning, chromosomal localization, and functional characterization of a human liver $\mathrm{Na}+$ bile acid cotransporter. J Clin Invest 93: 1326-1331, 1994.

2. Shiao T, Iwahashi M, Fortune J, Quattrochi L, Bowman S, Wick M, Qadri I and Simon FR: Structural and functional characterization of liver cell-specific activity of the human sodium/taurocholate cotransporter. Genomics 69: 203-213, 2000.

3. Anwer MS and Stieger B: Sodium-dependent bile salt transporters of the SLC10A transporter family: More than solute transporters. Pflugers Arch 466: 77-89, 2014.

4. Hagenbuch B and Dawson P: The sodium bile salt cotransport family SLC10. Pflugers Arch 447: 566-570, 2004.

5. Ho RH, Leake BF, Roberts RL, Lee W and Kim RB: Ethnicity-dependent polymorphism in $\mathrm{Na}$-taurocholate cotransporting polypeptide (SLC10A1) reveals a domain critical for bile acid substrate recognition. J Biol Chem 279: 7213-7222, 2004.

6. Pan W, Song IS, Shin HJ, Kim MH, Choi YL, Lim SJ, Kim WY, Lee SS and Shin JG: Genetic polymorphisms in $\mathrm{Na}^{+}$-taurocholate co-transporting polypeptide (NTCP) and ileal apical sodium-dependent bile acid transporter (ASBT) and ethnic comparisons of functional variants of NTCP among Asian populations. Xenobiotica 41: 501-510, 2011.

7. Shneider BL, Fox VL, Schwarz KB, Watson CL, Ananthanarayanan $\mathrm{M}$, Thevananther S, Christie DM, Hardikar W, Setchell KD, Mieli-Vergani G, et al: Hepatic basolateral sodium-dependent-bile acid transporter expression in two unusual cases of hypercholanemia and in extrahepatic biliary atresia. Hepatology 25: 1176-1183, 1997.

8. Vaz FM, Paulusma CC, Huidekoper H, de Ru M, Lim C, Koster J, Ho-Mok K, Bootsma AH, Groen AK, Schaap FG, et al: Sodium taurocholate cotransporting polypeptide (SLC10A1) deficiency: Conjugated hypercholanemia without a clear clinical phenotype. Hepatology 61: 260-267, 2015.

9. Van Herpe F, Waterham HR, Adams CJ, Mannens M, Bikker H, Vaz FM and Cassiman D: NTCP deficiency and persistently raised bile salts: An adult case. J Inherit Metab Dis 40: 313-315, 2017

10. Qiu JW, Deng M, Cheng Y, Atif RM, Lin WX, Guo L, Li H and Song YZ: Sodium taurocholate cotransporting polypeptide (NTCP) deficiency: Identification of a novel SLC10A1 mutation in two unrelated infants presenting with neonatal indirect hyperbilirubinemia and remarkable hypercholanemia. Oncotarget 8: 106598-106607, 2017.

11. Liu R, Chen C, Xia X, Liao Q, Wang Q, Newcombe PJ, Xu S, Chen M, Ding Y, Li X, et al: Homozygous p.Ser267Phe in SLC10A1 is associated with a new type of hypercholanemia and implications for personalized medicine. Sci Rep 7: 9214, 2017.

12. Deng M, Mao M, Guo L, Chen FP, Wen WR and Song YZ: Clinical and molecular study of a pediatric patient with sodium taurocholate cotransporting polypeptide deficiency. Exp Ther Med 12: 3294-3300, 2016.

13. Song YZ and Deng M: Sodium taurocholate cotransporting polypeptide deficiency manifesting as cholestatic jaundice in early infancy: A complicated case study. Zhongguo Dang Dai Er Ke Za Zhi 19: 350-354, 2017 (In Chinese). 
14. Li H, Qiu JW, Lin GZ, Deng M, Lin WX, Cheng Y and Song YZ: Clinical and genetic analysis of a pediatric patient with sodium taurocholate cotransporting polypeptide deficiency. Zhongguo Dang Dai Er Ke Za Zhi 20: 279-284, 2018 (In Chinese).

15. Tan HJ, Deng M, Qiu JW, Wu JF and Song YZ: Monozygotic twins suffering from sodium taurocholate cotransporting polypeptide deficiency: A case report. Front Pediatr 6: 354, 2018.

16. Chen R, Deng M, Rauf YM, Lin GZ, Qiu JW, Zhu SY, Xiao XM and Song YZ: Intrahepatic cholestasis of pregnancy as a clinical manifestation of sodium-taurocholate cotransporting polypeptide deficiency. Tohoku J Exp Med 248: 57-61, 2019.

17. Mao F, Liu T, Hou X, Zhao H, He W, Li C, Jing Z, Sui J, Wang F, Liu X, et al: Increased sulfation of bile acids in mice and human subjects with sodium taurocholate cotransporting polypeptide deficiency. J Biol Chem 294: 11853-11862, 2019.

18. Adzhubei IA, Schmidt S, Peshkin L, Ramensky VE, Gerasimova A, Bork P, Kondrashov AS and Sunyaev SR: A method and server for predicting damaging missense mutations. Nat Methods 7: 248-249, 2010

19. Bacalhau M,Pratas J,Simoes M, Mendes C, Ribeiro C, Santos MJ, Diogo L, Macario MC and Grazina M: In silico analysis for predicting pathogenicity of five unclassified mitochondrial DNA mutations associated with mitochondrial cytopathies' phenotypes. Eur J Med Genet 60: 172-177, 2017.

20. Kumar P, Henikoff S and Ng PC: Predicting the effects of coding non-synonymous variants on protein function using the SIFT algorithm. Nat Protoc 4: 1073-1081, 2009.

21. Quang D, Chen Y and Xie X: DANN: A deep learning approach for annotating the pathogenicity of genetic variants. Bioinformatics 31: 761-763, 2015.

22. Richards S, Aziz N, Bale S, Bick D, Das S, Gastier-Foster J, Grody WW, Hegde M, Lyon E, Spector E, et al: Standards and guidelines for the interpretation of sequence variants: A joint consensus recommendation of the American college of medical genetics and genomics and the association for molecular pathology. Genet Med 17: 405-424, 2015.

23. Hicks S, Wheeler DA, Plon SE and Kimmel M: Prediction of missense mutation functionality depends on both the algorithm and sequence alignment employed. Hum Mutat 32: 661-668, 2011.

24. Nykamp K, Anderson M, Powers M, Garcia J, Herrera B, Ho YY, Kobayashi Y, Patil N, Thusberg J, Westbrook M, et al: Sherloc: A comprehensive refinement of the ACMG-AMP variant classification criteria. Genet Med 19: 1105-1117, 2017

25. Hagenbuch B and Meier PJ: The superfamily of organic anion transporting polypeptides. Biochim Biophys Acta 1609: 1-18, 2003.

26. Mooij MG, Schwarz UI, de Koning BA, Leeder JS, Gaedigk R, Samsom JN, Spaans E, van Goudoever JB, Tibboel D, Kim RB and de Wildt SN: Ontogeny of human hepatic and intestinal transporter gene expression during childhood: Age matters. Drug Metab Dispos 42: 1268-1274, 2014.

27. Ballatori N, Christian WV, Lee JY, Dawson PA, Soroka CJ, Boyer JL, Madejczyk MS and Li N: OSTalpha-OSTbeta: A major basolateral bile acid and steroid transporter in human intestinal, renal and biliary epithelia. Hepatology 42: 1270-1279, 2005.

28. Goodwin B, Jones SA, Price RR, Watson MA, McKee DD Moore LB, Galardi C, Wilson JG, Lewis MC, Roth ME, et al: A regulatory cascade of the nuclear receptors FXR, SHP-1, and LRH-1 represses bile acid biosynthesis. Mol Cell 6: 517-526, 2000.
29. Boyer JL, Trauner M, Mennone A, Soroka CJ,Cai SY, Moustafa T, Zollner G, Lee JY and Ballatori N: Upregulation of a basolateral FXR-dependent bile acid efflux transporter OSTalpha-OSTbeta in cholestasis in humans and rodents. Am J Physiol Gastrointest Liver Physiol 290: G1124-G1130, 2006.

30. Frankenberg T, Rao A, Chen F, Haywood J, Shneider BL and Dawson PA: Regulation of the mouse organic solute transporter alpha-beta, Ostalpha-Ostbeta, by bile acids. Am J Physiol Gastrointest Liver Physiol 290: G912-G922, 2006.

31. Landrier JF, Eloranta JJ, Vavricka SR and Kullak-Ublick GA: The nuclear receptor for bile acids, FXR, transactivates human organic solute transporter-alpha and -beta genes. Am J Physiol Gastrointest Liver Physiol 290: G476-G485, 2006.

32. Tom Lissauer and Will Carroll: Illustrated textbook of paediatrics. Elsevier, Amsterdam, pp257, 2018.

33. Ignjatovic V, Lai C, Summerhayes R, Mathesius U, Tawfilis S, Perugini MA and Monagle P: Age-related differences in plasma proteins: How plasma proteins change from neonates to adults. PLoS One 6: e17213, 2011.

34. Robert M, Kliegman, Behrman, Jenson and Stanton: Nelson textbook of pediatrics. Saunders, Philadelphia, pp1660, 2007.

35. Suchy FJ, Balistreri WF, Heubi JE, Searcy JE and Levin RS: Physiologic cholestasis: Elevation of the primary serum bile acid concentrations in normal infants. Gastroenterology 80 : 1037-1041, 1981

36. Balistreri WF, Suchy FJ, Farrell MK and Heubi JE: Pathologic versus physiologic cholestasis: Elevated serum concentration of a secondary bile acid in the presence of hepatobiliary disease. J Pediatr 98: 399-402, 1981

37. Kawasaki H, Yamanishi Y, Miyake M, Mura T and Ikawa S Age- and sex-related profiles of serum primary and total bile acids in infants, children and adults. Tohoku J Exp Med 150: 353-357, 1986.

38. Sargiacomo C, El-Kehdy H, Pourcher G, Stieger B, Najimi M and Sokal E: Age-dependent glycosylation of the sodium taurocholate cotransporter polypeptide: From fetal to adult human livers. Hepatol Commun 2: 693-702, 2018.

39. Colombo C, Zuliani G, Ronchi M, Breidenstein J and Setchell KD: Biliary bile acid composition of the human fetus in early gestation. Pediatr Res 21: 197-200, 1987.

40. Colombo C, Roda A, Roda E, Buscaglia M, dell'Agnola CA, Filippetti P, Ronchi M and Sereni F: Correlation between fetal and maternal serum bile acid concentrations. Pediatr Res 19: 227-231, 1985.

41. Itoh S, Onishi S, Isobe K, Manabe M and Inukai K: Foetomaternal relationships of serum bile acid pattern estimated by high-pressure liquid chromatography. Biochem J 204: 141-145, 1982.

42. Monte MJ, Rodriguez-Bravo T, Macias RI, Bravo P, el-Mir MY, Serrano MA, Lopez-Salva A and Marin JJ: Relationship between bile acid transplacental gradients and transport across the fetal-facing plasma membrane of the human trophoblast. Pediatr Res 38: 156-163, 1995

This work is licensed under a Creative Commons Attribution-NonCommercial-NoDerivatives 4.0 International (CC BY-NC-ND 4.0) License. 